\title{
Fatores associados ao aleitamento materno exclusivo em Guarapuava, Paraná
}

\author{
Factors associated with exclusive breastfeeding in \\ Guarapuava, Paraná, Brazil
}

Marcela Komechen BRECAILO'

Arlete Catarina Tittoni CORSO²

Cláudia Choma Bettega ALMEIDA ${ }^{3}$

Bethsáida de Abreu Soares SCHMITZ ${ }^{4}$

RE S U M O

\section{Objetivo}

Esta pesquisa investigou a prevalência do aleitamento materno exclusivo aos seis meses de idade e sua associação com características socioeconômicas, demográficas, ambientais, de morbidade e biológicas.

\section{Métodos}

Foi realizado um estudo transversal e a amostra foi composta por 426 crianças entre zero e 23,9 meses, assistidas pelo Programa Saúde da Família e residentes na área urbana da cidade de Guarapuava (PR). Foram realizadas entrevistas domiciliares com a aplicação de questionários pré-codificados para investigar fatores relacionados ao aleitamento materno.

\section{Resultados}

A mediana de aleitamento materno exclusivo aos seis meses foi de sessenta dias e a prevalência foi de $12,9 \%$. Verificou-se associação estatisticamente significante $(p<0,05)$ do aleitamento materno exclusivo aos seis meses de idade com o trabalho materno fora do lar e com a continuidade do aleitamento materno até o momento da entrevista.

\section{Conclusão}

Os resultados indicam baixas prevalências de aleitamento materno exclusivo, além da necessidade de ações mais efetivas de amparo à mãe trabalhadora, que possui maiores dificuldades para a manutenção desta prática.

Termos de indexação: Aleitamento materno. Programa saúde da família. Prevalência. Trabalho feminino.

\footnotetext{
1 Artigo elaborado a partir da dissertação de M.K. BRECAILO, intitulada "Aleitamento materno: estudo com crianças menores de dois anos atendidas pelo programa saúde da família no município de Guarapuava - PR". Universidade Federal de Santa Catarina; 2007. Apoio: Coordenação de Aperfeiçoamento de Pessoal de Nível Superior (bolsa de mestrado).

2 Universidade Federal de Santa Catarina, Centro de Ciências da Saúde, Programa de Pós-Graduação em Nutrição, Departamento de Nutrição. Campus de Trindade, 88040-900, Florianópolis, SC, Brasil. Correspondência para/Correspondence to: A.C.T. CORSO.E-mail: <arlete@ccs.ufsc.br>.

3 Universidade Federal do Paraná. Paraná, Brasil.

${ }^{4}$ Universidade de Brasília, Departamento de Nutrição. Brasília, DF, Brasil.
} 
554 M.K. BRECAILO et al.

\section{A B S T R A C T}

\section{Objective}

This research investigated the prevalence of exclusive breastfeeding at the age of six months and its association with socioeconomic, demographic, environmental, biological and morbidity characteristics.

\section{Methods}

A cross-sectional study was done with a sample of 426 children from Guarapuava, PR, aged 0 to 23.9 months seen by the Family Health Program. Pre-coded questionnaires were administered during home interviews to investigate breastfeeding-associated factors.

\section{Results}

The median of exclusive breastfeeding at six months was 60 days and the prevalence was 12.9\%. Exclusive breastfeeding at six months was significantly associated $(p<0.05)$ with the mother's employment status and with breastfeeding continuing until the occasion of the interview.

\section{Conclusion}

The results indicate low prevalences of exclusive breastfeeding and the need of more effective actions to support working mothers, since it is harder for them to continue exclusive breastfeeding once they go back to work.

Indexing terms: Breast feeding. Family health program. Prevalence. Woman working.

\section{N T R O D U ÇÃ O}

O aleitamento materno é um dos fatores preponderantes na alimentação das crianças menores de dois anos de idade. Inúmeras são as vantagens para a mãe e para a criança. Para a mãe, há uma possível proteção contra câncer de mama e ovário, e para a criança, os principais benefícios incluem a proteção das vias respiratórias e do trato gastrointestinal contra doenças infecciosas. O leite materno promove ganho de peso adequado, é livre de contaminação, promovendo proteção imunológica, e estimula o vínculo afetivo entre mãe e filho' ${ }^{1}$.

Com o objetivo de uma melhor padronização dos termos utilizados em relação a esta prática, a Organização Mundial da Saúde (OMS) definiu diferentes categorias de aleitamento materno, sendo que as mesmas foram adotadas pelo Ministério da Saúde ${ }^{2}$. Neste sentido, entende-se que o Aleitamento Materno Exclusivo (AME) é aquele em que a criança recebe apenas leite humano, diretamente da mama ou ordenhado, de sua própria mãe ou de uma doadora, e nenhum outro líquido ou alimento sólido, com exceção de gotas ou xaropes de vitaminas, suplementos minerais ou medicamentos ${ }^{2}$.
O leite materno é capaz de suprir as necessidades da criança até os seis meses, e após essa idade deve ser complementado com alimentos adequados para atender as necessidades nutricionais e para prevenir a morbimortalidade infantil ${ }^{3}$. Verifica-se que a prática de aleitamento materno é capaz de prevenir 13\% das mortes de crianças menores de cinco anos, sendo uma das ações que mais contribui para a prevenção da mortalidade infantil ${ }^{4}$. Portanto, a OMS recomenda o aleitamento materno exclusivo nos primeiros seis meses de vida e o aleitamento materno até os dois anos ou mais 5 .

Resultados de pesquisas indicam que a introdução dos alimentos complementares antes do tempo recomendado pode induzir o desmame precoce. Esta prática pode estar associada a múltiplos fatores, como a atuação dos serviços de saúde, a escolaridade materna, a classe socioeconômica, o retorno precoce das nutrizes ao trabalho, o uso da chupeta, a ausência do pai, a gravidez precoce, as cesarianas eletivas, a separação precoce de mãe-bebê após o parto, a introdução de outros leites, o baixo peso ao nascer, e as crenças culturais ${ }^{6,7}$.

O Ministério da Saúde (MS) estimula a promoção, proteção e apoio ao aleitamento ma- 
terno por meio de alguns programas e projetos em crescente processo de expansão e qualificação, tornando-se imprescindível o acompanhamento sistemático das atividades relacionadas a essa ação.

Neste contexto, o Programa Saúde da Família (PSF) foi criado em 1994 pelo Ministério da Saúde, para integrar os princípios do Sistema Único de Saúde com a comunidade, priorizando as ações de proteção e promoção à saúde dos indivíduos e da família de forma integral e contínua. Este programa destaca a assistência materno-infantil, que envolve a promoção e o manejo do aleitamento materno ${ }^{8}$.

Entende-se que a implantação de ações de incentivo ao aleitamento materno no PSF, com atuação efetiva de equipes de Agentes Comunitários de Saúde (ACS) em serviços domiciliares, representa melhores oportunidades para divulgação, apoio e promoção do aleitamento materno, contribuindo para a intensificação desta prática entre crianças menores de seis meses ${ }^{9}$.

De acordo com Rea \& Toma ${ }^{10}$, apesar do grande número de pesquisas publicadas sobre a importância da prática de aleitamento materno exclusivo e complementar, ainda existe resistência em relação a essa temática, como se a mesma fosse uma prática simples e de estudo pouco importante. Se fazem necessárias novas pesquisas sobre a prática de aleitamento materno no país, demonstrando as tendências temporais sobre a mesma, uma vez que a última pesquisa de cunho nacional, foi realizada em 1999. Semelhantemente, é necessária a identificação das variáveis de maior preponderância para o efetivo sucesso desta ação, para que melhorias nesta prática possam ser alcançadas de forma mais contundente.

Nesse contexto, essa pesquisa faz parte de um projeto mais amplo intitulado "Promoção do aleitamento materno e alimentação complementar: um estudo de intervenção randomizado", tendo como objetivo geral verificar a prevalência de aleitamento materno exclusivo aos seis meses de idade e sua associação com as características socioeconômicas, demográficas, ambientais e de morbidade e biológicas, das famílias assistidas pelo PSF na cidade de Guarapuava, no estado do Paraná.

\section{M É T O D O S}

Foi realizado um estudo transversal em 25 Centros de Saúde, situados na área urbana da cidade de Guarapuava, município do centro-sul paranaense, localizado a $219 \mathrm{~km}$ de Curitiba. Em 2000, o município foi considerado como uma região de médio desenvolvimento pelo Índice de Desenvolvimento Humano. A taxa de urbanização na cidade é de $91,3 \%$, maior que a média do Estado. Observa-se que $80,0 \%$ da participação do Produto Interno Bruto (PIB) municipal são provenientes da indústria e setor de serviços e 20,0\% do setor agropecuário ${ }^{11}$.

Foram incluídas crianças de zero a 23,9 meses e suas mães biológicas, assistidas pelo PSF. O programa entrou em vigor neste município, em 2002 e desde então, vem crescendo continuamente. A cobertura em 2002 foi de $20,0 \%$, tendo passado para $65,0 \%$ em $2005^{11}$.

Para a seleção da amostra, foi considerado o total de 2454 crianças situadas na área urbana do município, assistidas no ano de 2004 pelo PSF em 25 Centros de Saúde, e, no momento da pesquisa, registradas pelos Agentes Comunitários de Saúde. Considerou-se $z=1,96$ - prevalência do fenômeno sob investigação, $(P)=0,5 \%$ ou $50 \%$ (por ser desconhecida a prevalência do fenômeno na população) e erro amostral previsto $(d)=5,0$ pontos percentuais para mais ou para menos (com 95\% IC), obtendo-se um número de 332 crianças. Após, estimou-se o efeito do desenho de 1,2 chegando-se a 399 crianças. Foram acrescidos $30 \%$ na amostra a fim de compensar eventuais perdas ou recusas por parte das mães em participar, totalizando 519 crianças.

Dentre as crianças que fizeram parte da amostra, foram excluídas as crianças gêmeas, as que não se encontravam sob os cuidados das mães biológicas, as que completaram dois anos 
antes da realização da entrevista e as que foram visitadas por três vezes sem que se conseguisse contato com a mãe. Os dados foram coletados nos domicílios das famílias previamente selecionadas, que recebiam a visita dos agentes comunitários de saúde.

Após consentirem em participar da pesquisa e assinarem o termo de consentimento livre e esclarecido, as mães das 519 crianças selecionadas na amostra, foram entrevistadas em suas casas por uma equipe previamente treinada.

As variáveis independentes selecionadas para esta pesquisa foram agrupadas em: Variáveis socioeconômicas: estado civil da mãe, escolaridade da mãe e do pai, trabalho materno remunerado, renda mensal familiar e recebimento de orientação sobre aleitamento materno. Variáveis demográficas: idade materna, número de filhos vivos atualmente e idade materna no nascimento do primeiro filho. Variáveis ambientais: número de pessoas no domicílio, tipo de domicílio, número de cômodos e número de dormitórios no domicílio, aglomeração no domicílio, presença de água encanada, presença de esgotamento sanitário ligado à rede pública e presença de coleta pública regular de lixo. Variáveis de morbidade e biológicas das famílias e das crianças: tipo de parto, peso ao nascer, presença de diarreia no dia ou na semana anterior à visita, presença de tratamento efetivo para a diarreia, interrupção da alimentação para a diarreia, presença de sintomas de doença respiratória, encaminhamento à consulta frente à tosse, internamento da criança nos últimos 12 meses, presença de aleitamento materno atualmente e gravidez atual. A variável dependente foi a prática de aleitamento materno exclusivo aos seis meses de idade.

Como instrumento de coleta de dados foi construído um questionário pré-codificado. Foi realizado um teste piloto com 82 mães que não participariam da pesquisa, para ajuste do referido questionário.

Os dados foram digitados em dupla entrada no Software Epi Info 6.04 e, posteriormente, foram exportados para o programa Stata 9.1 para as análises. Em um primeiro momento foram realizadas análises estatísticas descritivas: medidas de tendência central (média e mediana) e medida de dispersão (Desvio-Padrão - DP). Posteriormente foi realizado teste de associação por meio do qui-quadrado entre a variável dependente e as variáveis independentes. Todas as variáveis que apresentaram valor de $p<0,25$ no teste do qui-quadrado $\left(\chi^{2}\right)$ foram selecionadas para a análise de regressão multivariada não condicional. Foram estimadas as razões de chances (Odds Ratio), com respectivos intervalos de confiança de $95 \%$, brutos e ajustados. Foi utilizada a técnica de análise de sobrevivência para o cálculo da duração mediana do aleitamento materno.

A pesquisa foi aprovada pelo Comitê de Ética em Pesquisa do Centro de Saúde (CCS) Unicentro-UFPR, parecer $n^{\circ} 1$ 760/2006-15/03/06.

\section{RESULTA D OS}

Das 519 crianças inicialmente sorteadas, foram coletados dados de 426 , obtendo-se uma taxa de não resposta de $17,9 \%$. Ressalta-se que no cálculo da amostra foram acrescidos 30,0\% a fim de compensar eventuais perdas ou recusas. As entrevistas foram realizadas somente com as mães das crianças. Dentre as crianças que participaram da pesquisa 232 (54,5\%) eram do sexo masculino e 194 (45,5\%) do sexo feminino. A média de idade das crianças foi de 13,3 meses $(D P=5,8)$, sendo que o número de crianças maiores de um ano foi mais elevado do que o das menores de um ano. Apenas 13,1\% eram crianças menores de seis meses. As mães apresentaram idade média de 25,6 anos ( $D P=6,6$ ). A média de anos de estudo das mães foi de 7,1 anos ( $D P=3,5)$ e a dos pais foi de 7,0 ( $D P=3,3)$.

Dentre as famílias entrevistadas 97,9\% recebiam os serviços públicos de água encanada e 93,4\% a coleta pública regular de lixo, porém, o esgotamento sanitário foi constatado somente em $54,9 \%$ das famílias. Com relação aos dados socioeconômicos das famílias das crianças, verificou-se que $41,8 \%$ da população residiam em 
domicílios com cerca de cinco pessoas, os quais apresentavam em média 4,6 (DP=1,9) cômodos e 1,8 ( $D P=0,8)$ dormitórios, com uma densidade de 4,6 ( $\mathrm{DP}=1,8)$ pessoas por domicílio. Mais da metade dos domicílios $(57,5 \%)$ contava com dormitórios para três pessoas ou mais. A renda média familiar mensal foi de $\mathrm{R} \$ 623,48$ (DP $435,19)$. A renda média per capita mensal foi de $R \$ 151,75$ ( $D P=120,00)$, representando quase a metade do valor médio referido para a população de Guarapuava, que é de $R \$ 292,11^{12}$. Observou-se que 39,4\% das famílias viviam abaixo da linha da pobreza, ou seja, menos de 0,25 salários-mínimos por pessoa por mês de acordo com dados do Instituto Brasileiro de Geografia e Estatística (IBGE) de 2007 e 1,9\% das famílias não possuía renda no momento da entrevista.

O aleitamento materno apresentou média de 188,9 dias ( $\mathrm{DP}=144,6)$ e mediana de 180 dias, sendo que $49,7 \%$ das crianças ainda estavam recebendo aleitamento materno no momento da entrevista, não sendo, portanto, consideradas no cálculo de média e mediana por ainda não terem passado pelo processo de desmame.

O aleitamento materno exclusivo apresentou média de 74,6 dias ( $D P=62,0)$ e mediana de sessenta dias, sendo que $2,4 \%$ das crianças nunca receberam aleitamento materno. Observou-se que $97,4 \%$ receberam aleitamento materno de forma exclusiva após o nascimento, 74,9\% estavam em aleitamento materno exclusivo no primeiro mês, $43,9 \%$ no terceiro mês, $31,2 \%$ no quarto mês e apenas $12,9 \%$ das crianças receberam aleitamento materno exclusivo até os seis meses de idade ou mais, sem ultrapassar os dez meses de idade, segundo a recomendação da World Health Organization ${ }^{5}$ (Figura 1).

Comparando-se o aleitamento materno exclusivo segundo diferentes categorias de trabalho materno, observou-se (Figura 2) que as mães que trabalham fora de casa interrompem o aleitamento materno exclusivo mais precocemente do que as demais. As mães que possuem um trabalho remunerado dentro de casa amamentam de forma exclusiva seus filhos até o sexto mês com uma prevalência de $21,9 \%$, superior, portanto, às mães que trabalham fora de casa e as que não possuem trabalho remunerado.

As variáveis que apresentaram valor de $p<0,25$ no teste do qui-quadrado e que foram selecionadas para a análise de regressão multivariada não condicional foram a presença de aleitamento materno atualmente, a presença de coleta pública regular de lixo, a presença de água encanada, o trabalho materno remunerado, a escolaridade da mãe, a escolaridade do pai, a gravidez atual e o recebimento de orientação sobre aleitamento materno.

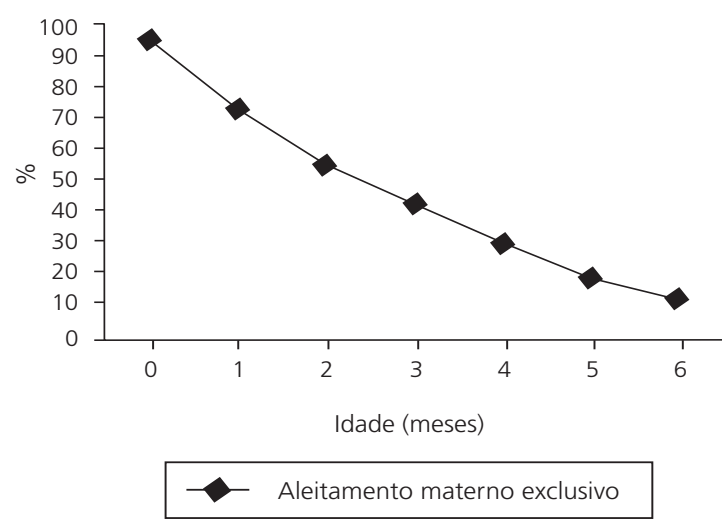

Figura 1. Crianças em aleitamento materno exclusivo de acordo com a idade em meses. Guarapuava (PR), 2007.

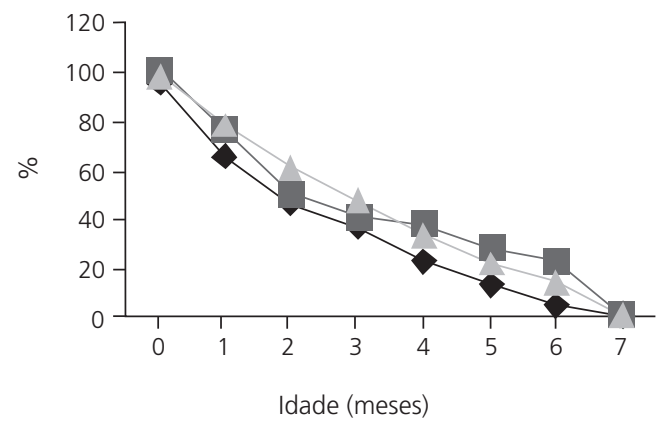

—-Mães que possuem trabalho remunerado fora de casa - Mães que possuem trabalho remunerado dentro de casa - Mães que não possuem trabalho remunerado

Figura 2. Crianças em aleitamento materno exclusivo por categorias de trabalho materno. Guarapuava (PR), 2007. 
Na Tabela 1 são apresentados os resultados da análise de regressão multivariada não condicional para todas as variáveis que apresentaram valor de $p<0,25$.

A associação entre aleitamento materno exclusivo aos seis meses e o trabalho materno fora de casa permaneceu significante $(p=0,04)$, sendo que mães que trabalham fora de casa possuem 3,92 vezes mais chances de não amamentarem exclusivamente seus filhos até os seis meses $(\mathrm{OR}=3,92$ e IC95\% 1,06-14,53).

A presença de aleitamento materno atualmente também permaneceu associada $(p=0,04)$ com o aleitamento materno exclusivo aos seis meses. Verifica-se, portanto, a tendência de haver continuidade do aleitamento materno até os dois anos de idade da criança, quando a mãe amamenta exclusivamente até os seis meses $(\mathrm{OR}=2,04$ e IC 95\% 1,03-4,01).

\section{I S C U S S Ã O}

A situação socioeconômica das famílias das crianças entrevistadas é precária em termos de renda mensal e de escolaridade dos pais. Por outro lado, observam-se condições mais favoráveis em

Tabela 1. Estimativa de odds ratio (OR) brutas e ajustadas e respectivos intervalos de confiança de $95 \%$ (IC $95 \%$ ) para a associação das variáveis aleitamento materno exclusivo, socioeconômicas, ambientais e de morbidade e biológicas de crianças menores de seis meses e suas mães biológicas assistidas pelo Programa Saúde da Família da cidade de Guarapuava (PR), 2007.

\begin{tabular}{|c|c|c|c|c|}
\hline Variáveis & Análise bruta (IC 95\%) & $p$-valor & Análise ajustada (IC 95\%) & $p$-valor \\
\hline \multicolumn{5}{|c|}{ Presença de aleitamento materno atualmente } \\
\hline Não & $2,10(1,16-3,80)$ & 0,015 & $2,04(1,03-4,01)$ & 0,04 \\
\hline Sim & 1,00 & & 1,00 & \\
\hline \multicolumn{5}{|c|}{ Presença de coleta pública regular de lixo } \\
\hline Não & $0,32(0,13-0,80)$ & $0,015^{*}$ & $0,54(0,18-1,57)$ & 0,25 \\
\hline Sim & 1,00 & & 1,00 & \\
\hline \multicolumn{5}{|l|}{ Presença de água encanada } \\
\hline Não & $0,18(0,04-0,86)$ & $0,047^{*}$ & $0,34(0,05-2,26)$ & 0,26 \\
\hline Sim & 1,00 & & 1,00 & \\
\hline \multicolumn{5}{|c|}{ Trabalho materno remunerado } \\
\hline Trabalho fora de casa & $3,53(1,22-10,14)$ & $0,008^{*}$ & $1,92(1,06-14,53)$ & 0,04 \\
\hline Trabalho dentro de casa & $0,60(0,24-1,48)$ & 0,26 & $0,48(0,19-1,23)$ & 0,13 \\
\hline Sem trabalho remunerado & 1,00 & & 1,00 & \\
\hline \multicolumn{5}{|l|}{ Escolaridade da mãe } \\
\hline 0 a 4 anos de estudo & $0,75(0,37-1,49)$ & 0,40 & $0,81(0,30-2,20)$ & 0,69 \\
\hline 5 a 8 anos de estudo & $0,60(0,28-1,26)$ & 0,18 & $0,99(0,41-2,39)$ & 0,98 \\
\hline$\geq 9$ anos de estudo & 1,00 & & 1,00 & \\
\hline \multicolumn{5}{|l|}{ Escolaridade do pai } \\
\hline 0 a 4 anos de estudo & $0,62(0,31-1,24)$ & 0,18 & $1,33(0,49-3,62)$ & 0,58 \\
\hline 5 a 8 anos de estudo & $0,99(0,44-2,23)$ & 0,99 & $0,80(0,33-1,91)$ & 0,61 \\
\hline$\geq 9$ anos de estudo & 1,00 & & 1,00 & \\
\hline \multicolumn{5}{|l|}{ Gravidez atual } \\
\hline Sim & $0,36(0,07-1,90)$ & $0,22^{*}$ & $0,33(0,06-1,64)$ & 0,17 \\
\hline Não & 1,00 & & 1,00 & \\
\hline \multicolumn{5}{|c|}{ Recebimento de orientação sobre aleitamento materno } \\
\hline Não & $0,65(0,32-1,31)$ & 0,23 & $0,65(0,29-1,45)$ & 0,29 \\
\hline Sim & 1,00 & & 1,00 & \\
\hline
\end{tabular}

*Teste exato de Fisher. 
relação à existência dos serviços públicos de água e de coleta de lixo nos domicílios.

Inseridas neste contexto, 50,2\% das crianças menores de dois anos de idade não recebiam mais aleitamento materno e $2,4 \%$, nunca chegaram a recebê-lo, o que pode gerar consequências negativas para toda a vida. A prevalência de aleitamento materno exclusivo encontrada foi de $12,9 \%$ aos seis meses de idade, mostrando-se superior à encontrada no último inquérito nacional realizado em outubro de 1999 nas capitais brasileiras, que indicou para o país uma prevalência de 7,7\%, e para a Região Sul de 10,2\% ${ }^{13}$. Destaca-se que a Região Sul teve a prevalência mais elevada dentre as regiões brasileiras pesquisadas para as três faixas de idade ${ }^{13}$.

Algumas pesquisas pontuais têm sido conduzidas em cidades brasileiras, encontrando distintas e variadas prevalências de aleitamento materno exclusivo aos seis meses de idade ${ }^{14-18}$.

Ferreira et al. ${ }^{15}$ observaram que a prevalência de aleitamento exclusivo em menores de seis meses aumentou de 13,0\% em 1995 para 29,6\% em 2004. Gomes ${ }^{19}$ em pesquisa realizada na cidade de Guarapuava, durante a Campanha de Vacinação, verificou que $37,3 \%$ das crianças menores de seis meses estavam em aleitamento materno exclusivo. Bittencourt et al. ${ }^{16}$ verificaram que o aleitamento materno exclusivo alcançou apenas $31,3 \%$ das crianças menores de um mês, índice que diminuiu continuamente até chegar à prevalência de 3,8\% ao sexto mês. Venâncio et al..20 realizaram estudo em 84 municípios do estado de São Paulo e verificaram uma prevalência de aleitamento materno exclusivo aos quatro meses entre $0 \%$ e $45,0 \%$, sendo superior a $20,0 \%$ em apenas $32,0 \%$ dos municípios. Marques et al..$^{21}$ realizaram pesquisa em quatro municípios nordestinos, sendo observado que $99,0 \%$ das mães iniciam a amamentação, porém $72,0 \%$ delas introduzem água ou chá no primeiro dia de vida. Neste contexto, a prevalência de aleitamento materno exclusivo aos seis meses foi de $0,6 \%$.

Os vários resultados apresentados, indicam a gravidade da situação desta prática no país. Em relação à mediana de aleitamento materno exclusivo, na cidade de Guarapuava, a mesma foi de 60 dias, mostrando-se superior à média nacional que é de 24 dias, e a média do Sul do Brasil, que é de 39 dias $^{1}$. Cecchetti \& Moura ${ }^{14}$ em Campinas (SP) indicaram mediana de aleitamento materno exclusivo de 67 dias. Outras pesquisas relataram medianas menos elevadas variando entre zero e 53,3 dias $15,17,18,22$.

Verifica-se, portanto, que a situação do aleitamento materno exclusivo na cidade de Guarapuava como em outras regiões do Brasil, não atinge as metas propostas pela OMS. Na cidade de Guarapuava, embora as mães estejam sendo assistidas pelo PSF, não se verificou um bom desempenho destes indicadores. Entende-se que, além do PSF, outros fatores e ações são relevantes para a promoção, proteção e apoio ao aleitamento materno. Entretanto, deve-se destacar que existem, no contexto e na estruturação das ações do PSF, condições potenciais efetivas de estímulo e acompanhamento do desempenho das práticas de aleitamento materno exclusivo, considerando-se a relevância do mesmo para a viabilização dos serviços na atenção materno-infantil.

Os resultados das pesquisas realizadas até o momento, reforçam a necessidade de melhorias na atenção básica, especialmente no PSF, de forma a capacitar e estimular estes profissionais, envolvendo a rede pública de saúde de forma mais efetiva, conduzindo assim a uma crescente melhora dos indicadores de aleitamento materno nas diversas regiões do País.

Nessa pesquisa, as variáveis que permaneceram associadas ao aleitamento materno exclusivo aos seis meses no modelo de regressão logística multivariada foram o trabalho materno fora de casa $(p=0,04)$ e a presença do aleitamento materno atualmente $(p=0,04)$.

O trabalho materno remunerado fora de casa vem se apresentando como um fator agravante para a interrupção do aleitamento materno exclusivo. No município de Guarapuava o mesmo é fator preponderante para a menor expectativa de duração do aleitamento materno exclusivo 
entre crianças menores de seis meses assistidas pelo PSF. Observou-se que o risco de interrupção do aleitamento materno exclusivo por mães que trabalham fora de casa foi 3,92 (OR=3,92; IC95\% $1,06-14,53)$ vezes mais elevado quando comparado com as mães que não trabalham fora de casa. Nesta população também foi visto que as mães que trabalham de forma remunerada em sua própria casa, amamentam exclusivamente seus filhos até os seis meses com prevalência mais elevada do que as mães que não trabalham, e do que as mães que trabalham fora de casa $(21,9 \%$ comparando-se com $14,4 \%$ e $4,5 \%$ respectivamente). Neste contexto, a existência de uma cobertura adequada de aparato social e legal de proteção à amamentação, como a licença maternidade e a presença de creches no ambiente de trabalho, entre outros, pode representar um fator de estímulo à manutenção da amamentação.

Em uma pesquisa realizada no estado do Rio de Janeiro, Oliveira \& Camacho ${ }^{9}$ encontraram o dobro do risco de interrupção precoce do aleitamento materno exclusivo entre mães de crianças menores de seis meses que trabalham fora de casa. Semelhantemente, Mascarenhas et al. ${ }^{23}$ encontraram em Pelotas-RS, um maior risco de interrupção do aleitamento materno exclusivo devido ao trabalho materno, em crianças menores de três meses. Comparando o aleitamento materno exclusivo e seus determinantes em três países Latino-Americanos, Pérez-Escamilla et al. ${ }^{24}$ observaram que no Brasil a interrupção do aleitamento materno exclusivo estava associada ao trabalho materno e em Honduras e no México, o aleitamento materno exclusivo estava associado à pior situação socioeconômica.

Alguns outros estudos revelam esta associação de resultados entre as condições socioeconômicas, o aleitamento materno e as questões relacionadas ao trabalho materno ${ }^{22,25}$. Segundo Araújo \& Almeida ${ }^{26}$, a atuação profissional na assistência à prática de aleitamento materno não deve permanecer numa abordagem restrita ao campo da biologia, em detrimento das especificidades culturais e emocionais da mulher, deven- do-se levar em consideração, também, as questões socioeconômicas que induzem as mulheres à procura de trabalho fora de casa.

Estes resultados indicam a necessidade de um olhar mais aprofundado sobre a importância da licença maternidade, inclusive trazendo à discussão o período de duração da mesma, além de outras estratégias de proteção ao aleitamento materno, destacando-se o aleitamento exclusivo. Ações de apoio e subsídio à mãe trabalhadora, tanto na rede formal quanto na rede informal, devem ser vistas como importantes estratégias de apoio à amamentação, devendo ser discutidas junto à comunidade e a sociedade em geral. Neste contexto, estudo realizado no estado da Paraíba, encontrou uma prevalência de aleitamento materno exclusivo em crianças de zero a quatro meses de $22,4 \%$, sendo significantemente maior entre as mães que trabalhavam fora e tiveram a licença maternidade ${ }^{27}$.

As recomendações da Organização Internacional do Trabalho (OIT) na revisão de 2001, indicam aos países que a licença-maternidade seja de 14 semanas, havendo pausas remuneradas para a prática do aleitamento materno durante o trabalho ${ }^{28}$. No Brasil, esse benefício é de 120 dias (17 semanas), sendo que alguns municípios já adotaram seis meses e encontra-se em discussão nas instâncias governamentais um projeto de lei dessa natureza.

Os resultados sobre o trabalho materno apresentados indicam que a efetividade das ações de incentivo à prática de aleitamento materno, desenvolvidas no Programa Saúde da Família, deve levar em consideração o aumento da participação da mulher no mercado de trabalho, incluindo neste contexto, as que se encontram em processo de aleitamento materno. Dentre os benefícios trabalhistas relacionados com a proteção da maternidade, aqueles referentes à possibilidade de amamentar são os menos cumpridos, devendo consistir em uma das preocupações das autoridades de saúde ${ }^{10}$.

Com relação à associação entre o aleitamento materno exclusivo até os seis meses e a 
presença de aleitamento materno até o momento da entrevista, verifica-se a necessidade de um aprofundamento desta análise para verificar esta relação no município de Guarapuava.

A contribuição das ações das Unidades de Saúde (UBS) é imprescindível para o sucesso da promoção do aleitamento materno exclusivo. A literatura indica que programas de puericultura bem conduzidos aumentaram a prevalência do aleitamento materno exclusivo em algumas localidades ${ }^{6,29}$. O Programa Saúde da Família, por meio de seus profissionais, como os agentes comunitários de saúde treinados e motivados na temática da amamentação, pode ser uma forte estratégia de incentivo e apoio ao aleitamento materno exclusivo, contribuindo para a manutenção do mesmo até a idade preconizada, destacando-se que estas ações devem ter início durante o pré-natal ${ }^{30}$.

Porém, faz-se necessária uma maior investigação sobre as características de ação do PSF em relação a sua atuação na promoção do aleitamento materno, no contexto da família. Instrumentos de avaliação devem ser desenvolvidos e testados com este objetivo, contribuindo assim para ações futuras de melhorias no incentivo e envolvimento da família no apoio ao aleitamento materno. De acordo com uma pesquisa sobre o Impacto das Unidades de Saúde na duração do aleitamento materno exclusivo, apenas um quinto dos profissionais de saúde havia sido treinado em cursos teórico-práticos de 18 horas ou mais sobre as vantagens e o manejo das práticas de aleitamento materno. Também se verificou que muitas UBS apesar de terem normas escritas de prática de aleitamento materno, não cobriam todos os Dez Passos da iniciativa Unidade Básica Amiga da Amamentação9.

Os resultados desta pesquisa permitiram concluir que as prevalências de aleitamento materno exclusivo e de aleitamento materno complementado encontradas na cidade de Guarapuava, entre crianças menores de dois anos de idade pertencentes às famílias assistidas pelo Programa Saúde da Família, encontravam-se inferiores às recomendações da Organização Mundial da Saúde e do Ministério da Saúde.

O trabalho materno fora de casa mostrou-se associado ao aleitamento materno exclusivo aos seis meses, indicando que o fato da mãe exercer atividades remuneradas fora de casa dificulta e interrompe a continuidade do aleitamento materno exclusivo aos seis meses, demonstrando a necessidade real de ações de apoio à mãe trabalhadora.

O aleitamento materno exclusivo aos seis meses e a continuidade do aleitamento materno complementado até o momento da entrevista mostraram-se associados, sendo, porém, necessário um aprofundamento no tema.

Os resultados desta pesquisa sugerem que os serviços públicos de saúde, por meio de estratégias como o próprio PSF, podem envidar esforços no sentido de aprimorar os indicadores de aleitamento materno e, deste modo, contribuir para uma melhoria da qualidade de vida da população.

Em uma perspectiva mais ampla, verifica-se que no rol de ações e estratégias voltadas para a promoção, proteção e apoio ao aleitamento materno, os avanços que podem ser observados parecem ser devido a mobilizações diversas, que envolvem setores governamentais, não governamentais, profissionais de saúde, voluntários e organismos internacionais.

No Brasil, além do Programa Saúde da Família, algumas outras iniciativas como a existência da Norma Brasileira para Comercialização de Alimentos para Lactentes e Crianças de Primeira Infância, Bicos, Chupetas e Mamadeiras ${ }^{31}$, os Hospitais Amigo da Criança ${ }^{32,33}$ e a Iniciativa Unidade Básica Amiga da Amamentação ${ }^{34}$, têm representado, ao longo do tempo, opções de estratégias importantes para aumentar as taxas de aleitamento materno no país.

\section{OLABORADORES}

M.K. BRECAILO participou da elaboração do projeto, da coleta e análise dos dados e da redação do 
artigo. A.C.T. CORSO participou da elaboração do projeto, análise dos dados, redação, correção e revisão do artigo. C.C.B. ALMEIDA participou da elaboração do projeto e da coleta dos dados e B.A.S. SCHMITZ participou da redação, correção e revisão do artigo.

\section{REFERÊ NCIAS}

1. Brasil. Ministério da Saúde. Secretaria de Política de Saúde. Organização Pan Americana da Saúde. Guia alimentar para crianças menores de dois anos. Brasília: Ministério da Saúde; 2002.

2. World Health Organization. Indicators for assessing breastfeeng practices. Geneva: WHO; 1991.

3. Monte CMG, Giuliani ERJ. Recomendações para alimentação complementar da criança em aleitamento materno. J Pediatr. 2004; 80(5):131-41.

4. Jones G, Steketee RW, Black RE, Bhutta ZA, Morris SS, Bellagio Child Survival Study Group. How many child deaths can we prevent this year? Lancet. 2003; 362(9377):65-71.

5. Word Health Organization. The optimal duration of exclusive brestfeeding: a systematic review. Geneva: WHO; 2001

6. Faleiros JJ, Kalil G, Casarin DP, Laque Jr PA, Santos IS. Avaliação do impacto de um programa de puericultura na promoção da amamentação exclusiva. Cad Saúde Pública. 2006; 21(2):482-9.

7. Saldiva SRDM, Escuder MM, Mondini L, Levy RB, Venancio SI. Práticas alimentares de crianças de 6 a 12 meses e fatores maternos associados. J Peditr. 2007; (83):53-8

8. Bonfim LA, Bastos ACS. O Impacto sociocultural do Programa Saúde da Família (PSF): uma proposta de avaliação. Cad Saúde Pública. 1998; 14(2): 429-35.

9. Oliveira MIC, Camacho LAB. Impacto das unidades básicas de saúde na duração do aleitamento materno exclusivo. Rev Bras Epidemiol. 2002; 5(1):41-51.

10. Rea MF, Toma TS. Amamentação: evidências científicas e ações para incentivar sua prática. In: Kac G, Sichieri R, Gigante DP, editores. Epidemiologia nutricional. Rio de Janeiro: Atheneu; 2007. v.1.

11. Conselho Nacional de Secretários Municipais de Saúde. Recortes do Brasil [Internet]. Curitiba; 2005 [acesso 2006 out 4]. Disponível em: <http://www. conasems.org.br/mostraPagina. asp?codServico= 1542\&codPagina $=2308$ > .

12. Programa das Nações Unidas para o Desenvolvimento. Atlas do desenvolvimento humano no Brasil [Internet]. Brasília; 2000 [acesso 2007 nov 28]. Disponível em: <http://www.pnud.org.br/atlas/>.
13. Brasil. Ministério da Saúde. Pesquisa de prevalência de aleitamento materno nas capitais e no Distrito Federal. Brasília: Ministério da Saúde; 2001.

14. Cecchetti DFA, Moura EC. Prevalência do aleitamento materno na região noroeste de Campinas, São Paulo, Brasil. Rev Nutr. 2005; 18(2):201-8. doi: 10.1590/S1415-52732005000200004.

15. Ferreira L, Parada CMGL, Carvalhães MABL. Tendência do aleitamento materno em município da região centro-sul do estado de São Paulo: 19951999-2004. Rev Nutr. 2007; 20(3):265-73. doi: 10.1590/S1415-52732007000300005.

16. Bittencourt LJ, Oliveira JS, Figueiroa JN, Batista Filho M. Aleitamento materno no estado de Pernambuco: prevalência e possível papel das ações de saúde. Rev Bras Saúde Matern Infant. 2005; 5(4): 439-48.

17. Chaves RG, Lamounier JA, Cesar CC. Factors associated with duration of breastfeeding. J Pediatr. 2007; 83(3):241-6.

18. Silva SM, Brunken GS, França GVA, Escuder MM, Venâncio SI. Evolução do aleitamento materno em uma capital da região centro-oeste do Brasil entre 1999 e 2004. Cad Saúde Pública. 2007; 23(7): $1539-46$.

19. Gomes PTT. Práticas alimentares de crianças menores de um ano vacinadas na segunda etapa da Campanha nacional de vacinação nos postos de saúde fixos de Guarapuava (PR) em 2004 [dissertação]. Ribeirão Preto: Universidade de São Paulo; 2005.

20. Venâncio SI, Escuder MML, Kitiko P, Rea MF, Monteiro CA. Freqüência e determinantes do aleitamento materno em municípios do Estado de São Paulo. Rev Saúde Pública. 2002; 36(3):313-8.

21. Marques NM, Lira PIC, Lima MC, Silva NL, Batista Filho M, Huttly SRA, et al. Breatfeeding and early weaning practices in Northeast Brazil: a longitudinal study. Pediatrics. 2001;108(4):1-9.

22. Vasconcelos MGL, Lira PIC, Lima MC. Duração e fatores associados ao aleitamento materno em crianças menores de 24 meses de idade no estado de Pernambuco. Rev Bras Saúde Matern Infant. 2006; 6(1):99-105.

23. Mascarenhas MLW, Albernaz EP, Silva MB, Silveira $\mathrm{RB}$. Prevalence of exclusive breastfeeding and its determiners in the first 3 months of live in the South of Brazil. J Pediatr. 2006; 8(4):289-94.

24. Pérez-Escamilla R, Lutter C, Segall AM, Treviño-Siller $\mathrm{S}$, Sanghvi T. Exclusive breast-feeding duration is associated with attitudinal, socioeconomic and biocultural determinants in three Latin American countries. J Nutr. 1995; 125:2972-84. 
25. Oliveira LPM, Assis AMO, Gomes GSS, Prado MS, Barreto ML. Duração do aleitamento materno, regime alimentar e fatores associados segundo condições de vida em Salvador, Bahia, Brasil. Cad Saúde Pública. 2005; 21(5):1519-30.

26. Araújo RMA, Almeida JAG. O aleitamento materno na pós-graduação no Brasil: um perfil das dissertações e teses de 1974 a 2004. Rev Bras Saúde Matern Infant. 2008; 8(1):125-33.

27. Vianna RPT, Rea MF, Venâncio SI, Escuder MM. A prática de amamentação entre mulheres que exercem trabalho remunerado na Paraíba, Brasil: um estudo transversal. Cad Saúde Pública. 2007; 23(10): 2403-9.

28. Word Alliance For Breastfeeding Action. Status of maternity prtection by country. [cited 2006 May 21]. Available from: <www.waba.org.my $>$.

29. Del Ciampo LA, Junqueira MJG, Ricco RG, Daneluzzi JC, Ferraz IS, Martinelli Jr. CE. Tendência secular do aleitamento materno em uma unidade de atenção primária à saúde materno-infantil em Ribeirão Preto, São Paulo. Rev Bras Saúde Mater Infant. 2006; 6(4):391-6.
30. Silveira RB, Albernaz E, Zuccheto LM. Fatores associados ao início da amamentação em uma cidade do Sul do Brasil. Rev Bras Saúde Matern Infant. 2008; 8(1):35-43.

31. Araújo MFM; Rea MF, Aragão K, Schmitz BAS. Avanços na norma brasileira de comercialização de alimentos para idade infantil. Rev Saúde Pública. 2006; 40(3):513-20.

32. Araújo MFM, Schmitz BAS. Evolução da Iniciativa Hospital Amigo da Criança no Brasil, entre 1992 e 2004. Rev Panam Salud Pública. 2007; 22:91-9.

33. Araújo MFM, Schmitz BAS. Reassessment of baby: friendly hospitals in Brazil. J Hum Lact. 2007; 23(3): 246-52.

34. Cardoso LO, Vicente AS, Damião JJ, Rito, RVVF. Impacto da implementação da Iniciativa Unidade Básica Amiga da Amamentação nas prevalências de aleitamento materno e nos motivos de consulta em uma unidade básica de saúde. J Pediatr. 2008; 84(2):147-53.

Recebido em: 16/9/2008

Versão final reapresentada em: 29/4/2009

Aprovado em: 11/11/2009 